\title{
A multidisciplinary journal focused on endocrine oncology
}

"Our goal in the development of this journal is to provide a high-impact venue for the many investigators and clinicians who have a focus on endocrine cancers."

Keywords: endocrine oncology $\bullet$ diagnosis $\bullet$ hormone $\bullet$ imaging $\bullet$ therapy

On behalf of our distinguished Editorial Board, we are delighted to welcome you to the inaugural edition of the International Journal of Endocrine Oncology, a multidisciplinary journal focused on endocrine malignancies. To our knowledge, this is the first journal to have the sole focus on endocrine cancers encompassing basic and translational science, and the clinical management of endocrine cancers. Our goal in the development of this journal is to provide a high-impact venue for the many investigators and clinicians who have a focus on endocrine cancers. We are seeking to encourage submissions from all stakeholders in endocrine cancers, including laboratory scientists, endocrinologists, medical oncologists, surgeons, radiologists and pathologists, among others.

The time is right to have a journal focused on endocrine malignancies (pituitary, parathyroid, adrenal, thyroid and pancreas) as the epidemiology of endocrine cancers, clinical management strategies and our understanding of the genetics/genomics have changed dramatically. Because of this, many leading academic institutions now have dedicated multidisciplinary clinical and or research programs in endocrine oncology. Given these changes in epidemiology, advances in therapy, and emerging questions and controversies, we believe 'the time is right' to have a journal focused on endocrine oncology. We believe this journal will have a broad readership given the many specialties involved in the management of endocrine malignancies. We welcome articles and special issue focus proposals to the editorial team, and we encourage you to submit your best work to the International Journal of Endocrine Oncology.

\section{Financial \& competing interests disclosure}

The authors have no relevant affiliations or financial involvement with any organization or entity with a financial interest in or financial conflict with the subject matter or materials discussed in the manuscript. This includes employment, consultancies, honoraria, stock ownership or options, expert testimony, grants or patents received or pending, or royalties.

No writing assistance was utilized in the production of this manuscript.

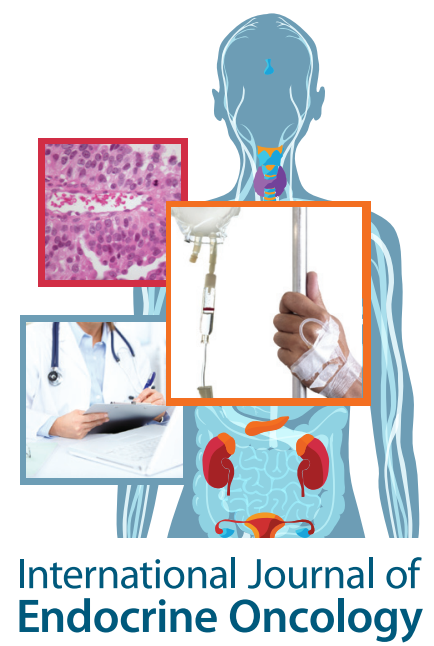

Electron Kebebew

Author for correspondence: Endocrine Oncology Branch, National Cancer Institute, Bethesda, MD 20892, USA

Tel.: +1 3014965049

Fax: +1 3014021788

kebebewe@mail.nih.gov

Karel Pacak

Program in Reproductive \& Adult Endocrinology, Eunice Kennedy Shriver National Institute of Child Health \& Human Development, Bethesda, MD 20892, USA 